\title{
Modeling the Adsorption of Rheology Modifiers onto Latex Particles using Coarse-Grained Molecular Dynamics (CG-MD) and Self-Consistent Field Theory (SCFT) - Supporting Information
}

\author{
Valeriy V. Ginzburg ${ }^{1 *}$, Antony Keith Van Dyk ${ }^{2}$, Tirtha Chatterjee ${ }^{1}$, Alan Isamu Nakatani ${ }^{2}$, Shihu Wang ${ }^{3}$, \\ Ronald G. Larson ${ }^{3}$
}

${ }^{1}$ The Dow Chemical Company, Midland MI 48674 USA

${ }^{2}$ The Dow Chemical Company, Collegeville PA 19477 USA

${ }^{3}$ Department of Chemical Engineering, University of Michigan, Ann Arbor MI 48109 USA

This supporting information describes the intra- and intermolecular potentials used in the simulation, as well as the equations of the Self-Consistent Field Theory. 


\section{Coarse-Grained Molecular Dynamics}

The non-bonded interactions between any two coarse-grained beads is described by a 6-12 Lennard-Jones $(\mathrm{LJ})$ potential $\mathrm{U}_{\mathrm{LJ}}$ :

$$
U_{L J}(r)=4 \epsilon\left[\left(\frac{\sigma}{r}\right)^{12}-\left(\frac{\sigma}{r}\right)^{6}\right]
$$

In this study, the LJ potential has a cutoff distance of $1.2 \mathrm{~nm}$ and is shifted gradually to zero from $0.9 \mathrm{~nm}$ to $12 \mathrm{~nm}$ using the standard shift function in GROMACS so that the potential and force are zero at the cutoff distance.

Bond is described by a weak harmonic stretching potential $\mathrm{U}_{\text {bond }}$ :

$$
U_{\text {bond }}(d)=\frac{1}{2} k_{b}\left(d-d_{0}\right)^{2}
$$

Angle potential $\mathrm{U}_{\text {angle }}$ is shown below:

$$
U_{\text {angle }}(\theta)=\frac{1}{2} k_{\theta}\left(\cos (\theta)-\cos \left(\theta_{0}\right)\right)^{2}
$$

Instead of using a dihedral potential, a piecewise linear/harmonic distance restraints $U_{r}$ is imposed on the separation of the $\mathrm{i}^{\text {th }}$ and $\mathrm{i}+3^{\text {rd }} \mathrm{EO}$ beads, as shown below:

$$
U_{r}\left(r_{i, i+3}\right)=\left\{\begin{array}{lr}
0 & r_{i, i+3}<r_{1} \\
0.5 k_{d r}\left(r_{i, i+3}-r_{1}\right)^{2} & r_{1} \leq r_{i, i+3}<r_{2} \\
0.5 k_{d r}\left(r_{2}-r_{1}\right)\left(2 r_{i, i+3}-r_{2}-r_{1}\right) & r_{2} \leq r_{i, i+3}
\end{array}\right.
$$

The parameters for both bonded and non-bonded potentials used in the simulation are summarized in Table 1 of the manuscript. 


\section{Self-Consistent Field Theory}

The free energy of the system is given by,

$$
\begin{aligned}
\frac{F}{\rho_{0} V k T}= & -n_{H E U R} \ln \left[\frac{Q_{\text {HEUR }}}{V \varphi_{H E U R}}\right]-n_{w} \ln \left[\frac{Q_{w}}{V \varphi_{w}}\right]- \\
& \frac{1}{V} \int \mathbf{d r}\left[W_{C}(\mathbf{r}) \phi_{C}(\mathbf{r})+W_{E}(\mathbf{r}) \phi_{E}(\mathbf{r})+W_{w}(\mathbf{r}) \phi_{w}(\mathbf{r})\right] \\
& \frac{1}{V} \int \mathbf{d r} \xi(\mathbf{r})\left[1-\phi_{w}(\mathbf{r})-\phi_{C}(\mathbf{r})-\phi_{E}(\mathbf{r})-\phi_{B}(\mathbf{r})\right]+ \\
& \frac{1}{V} \int \mathbf{d r}\left[\chi_{C E} \phi_{C}(\mathbf{r}) \phi_{E}(\mathbf{r})+\chi_{C w} \phi_{C}(\mathbf{r}) \phi_{w}(\mathbf{r})+\chi_{w E} \phi_{w}(\mathbf{r}) \phi_{E}(\mathbf{r})\right]+ \\
& \frac{1}{V} \int \mathbf{d r}\left[\chi_{B E} \phi_{B}(\mathbf{r}) \phi_{E}(\mathbf{r})+\chi_{B w} \phi_{B}(\mathbf{r}) \phi_{w}(\mathbf{r})+\chi_{B C} \phi_{B}(\mathbf{r}) \phi_{C}(\mathbf{r})\right]
\end{aligned}
$$

where $\phi_{B}(\mathbf{r}), \phi_{E}(\mathbf{r}), \phi_{C}(\mathbf{r})$, and $\phi_{w}(\mathbf{r})$ are local (spatially varying) volume fractions of binder (B), EOblock (E), hydrophobe (C), and water (W), respectively; $\varphi_{H E U R}$ and $\varphi_{W}$ are the overall volume fractions of HEUR and water; then number densities of HEUR and water molecules are $n_{\text {HEUR }}=\frac{\varphi_{H E U R}}{N_{H E U R}}, n_{w}=\frac{\varphi_{w}}{N_{w}}$. The single-molecule partition functions for HEUR and water are given by,

$$
\begin{aligned}
& Q_{\text {HEUR }}=\int_{V} d \mathbf{r}\left[q_{\text {HEUR }}(\mathbf{r}, 1)\right] \\
& Q_{W}=\int_{V} d \mathbf{r}\left[q_{W}\left(\mathbf{r}, \alpha_{W}\right)\right]
\end{aligned}
$$

where $\alpha_{W}=\frac{N_{W}}{N_{H E U R}}$. The Gaussian-chain forward propagators are determined using the standard modified diffusion equation (MDE) approach: 
$\frac{\partial q_{H E U R}(\mathbf{r}, s)}{\partial s}=\left[\nabla^{2}-w_{t(s)}(\mathbf{r})\right] q_{H E U R}(\mathbf{r}, s)$

$\frac{\partial q_{W}(\mathbf{r}, s)}{\partial s}=\left[\nabla^{2}-w_{W}(\mathbf{r})\right] q_{W}(\mathbf{r}, s)$

For HEUR triblocks, index $t(s)=C$ for the two end-block hydrophobes and $E$ for the middle block; the first end of the chain corresponds to $s=0$, and the other end corresponds to $s=1$. For water, $s$ runs between 0 and $\alpha_{W}$. The reverse propagators are defined in a similar fashion,

$\frac{\partial q_{H E U R}^{+}(\mathbf{r}, s)}{\partial s}=\left[\nabla^{2}-w_{t(s)}(\mathbf{r})\right] q_{H E U R}^{+}(\mathbf{r}, s)$

$\frac{\partial q_{W}^{+}(\mathbf{r}, s)}{\partial s}=\left[\nabla^{2}-w_{W}(\mathbf{r})\right] q_{W}^{+}(\mathbf{r}, s)$

The initial conditions for the MDEs are as follows: $q_{H E U R}(\mathbf{r}, 0)=q_{H E U R}^{+}(\mathbf{r}, 1)=q_{W}(\mathbf{r}, 0)=q_{W}^{+}\left(\mathbf{r}, \alpha_{W}\right)=1$ outside the binder particles and 0 inside the particles. The Laplacian operator $\nabla^{2}$ is based on the units of length where the HEUR radius of gyration $R_{g}=a\left(N_{\text {HEUR }} / 6\right)^{1 / 2}$ is set to unity.

The binder density, $\phi_{B}(\mathbf{r})$, is given by,

$\phi_{B}(\mathbf{r})=\phi_{B}(x, y, z)=\left[\begin{array}{c}1, \text { if } \mathrm{z}<\mathrm{Z}_{1} \text { or } \mathrm{z}>\mathrm{n}_{\mathrm{z}}-\mathrm{Z}_{1} \\ 0.5, \text { if } \mathrm{z}=\mathrm{Z}_{1} \text { or } \mathrm{z}=\mathrm{n}_{\mathrm{z}}-\mathrm{Z}_{1} \\ 0, \text { if } \mathrm{Z}_{1}<\mathrm{z}<\mathrm{n}_{\mathrm{z}}-\mathrm{Z}_{1}\end{array}\right.$

In our simulations, $n_{z}=120$, and $Z_{1}=10$.

The self-consistency equations for densities and fields are obtained by minimizing the free energy and are as follows,

$$
\begin{aligned}
& W_{W}(\mathbf{r})=\chi_{C W} N_{H E U R} \phi_{C}(\mathbf{r})+\chi_{E W} N_{H E U R} \phi_{E}(\mathbf{r})+\chi_{B W} N_{H E U R} \phi_{B}(\mathbf{r})+\xi(\mathbf{r}) \\
& W_{C}(\mathbf{r})=\chi_{C W} N_{H E U R} \phi_{W}(\mathbf{r})+\chi_{C E} N_{H E U R} \phi_{E}(\mathbf{r})+\chi_{C B} N_{H E U R} \phi_{B}(\mathbf{r})+\xi(\mathbf{r})
\end{aligned}
$$




$$
\begin{aligned}
& W_{E}(\mathbf{r})=\chi_{E W} N_{H E U R} \phi_{W}(\mathbf{r})+\chi_{C E} N_{H E U R} \phi_{C}(\mathbf{r})+\chi_{B E} N_{H E U R} \phi_{B}(\mathbf{r})+\xi(\mathbf{r}) \\
& \phi_{W}(\mathbf{r})+\phi_{C}(\mathbf{r})+\phi_{E}(\mathbf{r})+\phi_{B}(\mathbf{r})=1 \\
& \phi_{W}(\mathbf{r})=\frac{V}{Q_{W}} \int_{0}^{\alpha_{W}} d s\left[q_{W}^{+}(\mathbf{r}, s) q_{W}(\mathbf{r}, s)\right] \\
& \phi_{C}(\mathbf{r})=\frac{V}{Q_{H E U R}}\left[\int_{0}^{f} d s\left[q_{H E U R}^{+}(\mathbf{r}, s) q_{H E U R}(\mathbf{r}, s)\right]+\int_{1-f}^{1} d s\left[q_{H E U R}^{+}(\mathbf{r}, s) q_{H E U R}(\mathbf{r}, s)\right]\right] \\
& \phi_{C}(\mathbf{r})=\frac{V}{Q_{H E U R}}\left[\int_{f}^{1-f} d s\left[q_{H E U R}^{+}(\mathbf{r}, s) q_{H E U R}(\mathbf{r}, s)\right]\right]
\end{aligned}
$$

Here, we defined $f$ as a volume fraction of the end hydrophobe relative to the whole HEUR; note that there are two hydrophobes per chain, so the overall hydrophobe volume fraction is $2 f$. The selfconsistency equations are solved in an iterative fashion using Picard algorithm, until the solution is converged according to a prescribed criterion, as discussed in the main text. 